\title{
Resonant formation of strongly correlated paired states in rotating Bose gases
}

\author{
S. G. Bhongale, J. N. Milstein, and M. J. Holland \\ JILA, University of Colorado and National Institute of Standards and Technology, Boulder, Colorado 80309-0440
}

\begin{abstract}
We propose increasing the fractional quantum Hall gap of a rapidly rotating Bose gas by increasing the interatomic interactions via a Feshbach resonance. The generation of molecules by the resonance causes pair correlations to grow throughout the system effecting the ground state. By an extension of the usual Chern-Simons theory, built of composite atoms and molecules, we are able to account for these resonance effects. We find that the resulting ground state evolves from a Laughlin wavefunction to a unique paired wavefunction as one approaches the resonance.
\end{abstract}

PACS numbers: 03.75.-b,74.43.-f,71.27.+a

Recently, there has been considerable interest from both the experimental and theoretical perspective in the behavior of rotating Bose gases confined to an effective two-dimensional space [1, 2, 3, 4, 5, 6]. One goal has been to understand and to create strongly correlated states, such as the Laughlin state generated by the fractional quantum Hall effect (FQHE), within ultracold atomic gases. A major challenge to realizing such a state is the need to reach extremely low temperatures in order to resolve the lowest Laughlin state from the first excited state. The size of this energy gap, however, is directly related to the strength of interatomic interactions, so by increasing the interactions it would seem natural that one could increase the gap, making the system more accessible to experiment.

Atomic systems can now be created which allow the microscopic interactions to be dynamically tuned [7, 8]. Feshbach resonances have proven very successful at doing this, allowing one to tune the interactions by adjusting the resonant detuning $\nu$ (for example, by varying a magnetic field), and would seem an excellent tool for increasing the gap within a FQHE system. To account for the full effects of the resonance, however, we cannot simply scale the mean-field energy but must incorporate the entire resonant structure into our model. This means that we must include the process of molecular formation generated by the introduction of a bound state within the open channel of scattering states $[9,10,11$.

By introducing a bound state, however, we not only modify the relative interaction strength, but we also introduce a physical mechanism for generating pair correlations between particles (see Fig. (1). In the context of two-dimensional condensed matter systems, such a mechanism, although arising from a very different source [12], can have a significant effect on the ground state properties. Before we can study the resonant behavior of the gap, we must first understand the effect of resonant interactions on the ground state wavefunction. We will find that as we approach the Feshbach resonance the Laughlin state transforms into a unique, strongly correlated state.

We begin by writing down an effective Hamiltonian, in second quantized form, for a resonant gas of

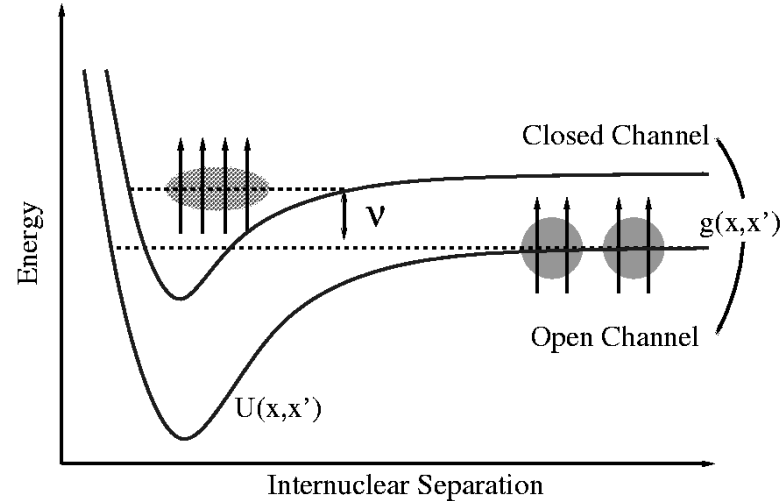

FIG. 1: The Feshbach resonance pairing mechanism is illustrated by the above Born-Oppenheimer curves. Pairs of composite atoms composed of single atoms and an associated number of quanta of angular momentum (represented by the arrows) approach each other within an open channel potential of background value $U\left(\mathbf{x}, \mathbf{x}^{\prime}\right)$. They may form a composite molecule due to the presence of a closed channel bound state, at a detuning $\nu$ from the scattering continuum, which is coupled to the open channel with strength $g\left(\mathbf{x}, \mathbf{x}^{\prime}\right)$.

Bosons of mass $m$ rotating in two dimensions with stirring frequency $\Omega$ approaching the trapping frequency $\omega$, i.e. $\omega-\Omega \rightarrow 0^{+}$:

$$
\begin{aligned}
\hat{H} & =\int d^{2} x \hat{\psi}_{a}^{\dagger}(\mathbf{x})\left[\frac{-1}{2 m}(\nabla-i \mathbf{A}(\mathbf{x}))^{2}\right] \hat{\psi}_{a}(\mathbf{x}) \\
& +\frac{1}{2} \int d^{2} x^{\prime} \int d^{2} x \hat{\psi}_{a}^{\dagger}(\mathbf{x}) \hat{\psi}_{a}^{\dagger}\left(\mathbf{x}^{\prime}\right) U\left(\mathbf{x}, \mathbf{x}^{\prime}\right) \hat{\psi}_{a}\left(\mathbf{x}^{\prime}\right) \hat{\psi}_{a}(\mathbf{x}) \\
& +\int d^{2} x \hat{\psi}_{m}^{\dagger}(\mathbf{x})\left[\frac{-1}{4 m}(\nabla-2 i \mathbf{A}(\mathbf{x}))^{2}+\nu\right] \hat{\psi}_{m}(\mathbf{x}) \\
& +\frac{1}{2} \int d^{2} x^{\prime} \int d^{2} x\left[\hat{\psi}_{m}^{\dagger}\left(\frac{\mathbf{x}+\mathbf{x}^{\prime}}{2}\right) g\left(\mathbf{x}, \mathbf{x}^{\prime}\right) \hat{\psi}_{a}(\mathbf{x}) \hat{\psi}_{a}\left(\mathbf{x}^{\prime}\right)+\text { H.c. }\right] .
\end{aligned}
$$

Here $\hat{\psi}_{a, m}^{\dagger}(\mathbf{x}), \hat{\psi}_{a, m}(\mathbf{x})$ are the creation and destruction operators for atoms and molecules which satisfy the commutation relations $\left[\hat{\psi}_{1}(\mathbf{x}), \hat{\psi}_{2}^{\dagger}\left(\mathbf{x}^{\prime}\right)\right]=\delta^{(3)}\left(\mathbf{x}, \mathbf{x}^{\prime}\right) \delta_{1,2}$, where $1,2 \in\{a, m\}$. We define the two-dimensional vector potential $\mathbf{A}(\mathbf{x})=(m \omega y,-m \omega x), U\left(\mathbf{x}, \mathbf{x}^{\prime}\right)$ is the twoparticle background scattering potential, $g\left(\mathbf{x}, \mathbf{x}^{\prime}\right)$ is the 
resonant coupling between the open and closed channel potentials, and $\nu$ is the detuning of the open channel continuum from the level of the bound state in the closed channel.

Recently, it has been noted that there is a direct mapping between the Hamiltonian for a rotating twodimensional gas, the first two terms of Eq. (1), and that of the FQHE [4]. The analog of the magnetic field is realized by the angular rotation and the Coulomb interaction is replaced by the two-particle scattering. Therefore, if we were to neglect resonant effects we would expect the many-body ground state to be described by the Laughlin wavefunction 13 .

$$
\Psi_{L}\left(\mathbf{x}_{1}, \mathbf{x}_{2}, \ldots \mathbf{x}_{N}\right)=\prod_{i<j}\left(z_{i}-z_{j}\right)^{2} \prod_{k} \exp \left(-\left|z_{k}\right|^{2} / 2\right),
$$

where the products run over the indices $i, j, k=$ $(1,2, \ldots N)$ at position $z=x+i y$ for $N$ particles. We will see that the inclusion of the resonant terms in Eq. (11) can significantly modify the form of Eq. (2) due to the growth of two-particle correlations.

We approach this problem by an extension of the Chern-Simons theory 14] which allows us to develop a mean-field theory for the rotating system that has removed the complications of the associated rotation. This is done by constructing a composite particle composed of the original particle and an artificially attached number of flux quanta. The composite particle is designed so that the attached flux quanta cancel the total rotation of the original system leaving a system of non-rotating, interacting composite particles. For the resonant system, the composite particles can formally be obtained by the following transformation:

$$
\begin{aligned}
\hat{\varphi}_{a, m}(\mathbf{x})=\exp [ & -q \int d^{2} x^{\prime} \theta\left(\mathbf{x}-\mathbf{x}^{\prime}\right) \\
& \left.\times\left[\rho_{a}\left(\mathbf{x}^{\prime}\right)+2 \rho_{m}\left(\mathbf{x}^{\prime}\right)\right]\right] \hat{\psi}_{a, m}(\mathbf{x}),
\end{aligned}
$$

where $\rho_{a}\left(\mathbf{x}^{\prime}\right)$ and $\rho_{m}\left(\mathbf{x}^{\prime}\right)$ are the atomic and molecular spatial densities, respectively, and $\theta\left(\mathbf{x}-\mathbf{x}^{\prime}\right)$ is the topological phase. To guarantee that the resulting composite particle is a Boson, with no loss of generality we set $q=2$.

In the composite picture, we replace the $\hat{\psi}$ operators in Eq. (II) with the corresponding composite operators $\hat{\varphi}$ and introduce the statistical Chern-Simons field $\mathbf{a}(\mathbf{x})$ through the gauge transformation

$$
\mathbf{A}(\mathbf{x}) \rightarrow \mathbf{A}(\mathbf{x})+\mathbf{a}(\mathbf{x})
$$

These modifications generate the Hamiltonian formulation of our composite atom/molecule theory. The composite picture can be shown to be equivalent to the single particle picture of Eq. (11).

We will now shift to a functional representation of the composite atom/molecule system to clarify the resonant modifications of the Chern-Simons theory and then return to the Hamiltonian formulation to derive the ground state wavefunction. Defining the action within one temporal and two spatial dimensions

$$
S=\int d^{3} x \sum_{\sigma=a, m} \varphi_{\sigma}^{*}(\mathbf{x}) i \partial_{0} \varphi_{\sigma}(\mathbf{x})-\int d x_{0} \hat{H}
$$

we generate a Chern-Simons term which couples to the statistical vector field $a_{\mu}(\mathbf{x})$

$$
S_{C S}=-\int d^{3} x \frac{1}{8 \pi} \epsilon^{\mu \nu \lambda} a_{\mu}(\mathbf{x}) \partial_{\nu} a_{\lambda}(\mathbf{x}),
$$

where the indices of $\mu, \nu$, and $\lambda$ run over the three dimensions $(0,1,2)$ and the summation convention over repeated indices is invoked. We have also introduced the antisymmetric tensor $\epsilon^{012}=1$. To simplify the following calculations we assume contact interactions of the form $U\left(\mathbf{x}, \mathbf{x}^{\prime}\right)=U \delta\left(\mathbf{x}, \mathbf{x}^{\prime}\right)$ and $g\left(\mathbf{x}, \mathbf{x}^{\prime}\right)=g \delta\left(\mathbf{x}, \mathbf{x}^{\prime}\right)$. Any complications arising from this replacement of the true potentials with contact potentials should be remedied as explained in Ref. [15]. We next perform the lowest order variation of the action.

Varying with respect to the zeroth component of the gauge field, $\partial S / \partial a_{0}=0$, reproduces the Chern-Simons condition

$$
\nabla \times\left.\mathbf{a}(\mathbf{x})\right|_{z}=-4 \pi\left(\left|\varphi_{a}(\mathbf{x})\right|^{2}+2\left|\varphi_{m}(\mathbf{x})\right|^{2}\right) .
$$

Equation (7) is a statement of Gauss's law for the statistical gauge field associating an even number of rotational flux quanta with each particle. This relation is simply a restatement of our choice of quasiparticle.

Since we will be interested in the ground state properties of the atom/molecule system, let us assume that the fields $\varphi_{a}(\mathbf{x})$ and $\varphi_{m}(\mathbf{x})$ are uniform. By minimizing the action with respect to the atomic and molecular fields, i.e. $\partial S / \partial \varphi_{a}=0, \partial S / \partial \varphi_{m}=0$, we generate the following constraint equation for the molecules:

$$
\varphi_{m}=\frac{g \varphi_{a}^{2}}{2\left[\nu+|\mathbf{A}+\mathbf{a}|^{2} / m\right]} .
$$

Equation (8) allows us to eliminate the molecular field from the theory and arrive at a self-consistent relationship for the gauge field

$$
|\mathbf{A}+\mathbf{a}|^{2}=\left(U+\frac{g^{2}}{4\left(\nu+|\mathbf{A}+\mathbf{a}|^{2} / m\right)}\right) 2 m\left|\varphi_{a}\right|^{2} .
$$


Equation (9) is the usual result relating the gauge field to the background density only now it is dependent upon the detuning from the resonance.

We now switch back to the Hamiltonian form of our theory to derive the ground state wavefunction. After Fourier transforming the composite form of Eq. (1) by substitution of the field operators $\hat{\varphi}_{a}(\mathbf{x})=\sum_{\mathbf{k}} \hat{a}_{\mathbf{k}} e^{i \mathbf{k} \cdot \mathbf{x}}$ and $\hat{\varphi}_{m}(\mathbf{x})=\sum_{\mathbf{k}} \hat{b}_{\mathbf{k}} e^{i \mathbf{k} \cdot \mathbf{x}}$, we follow the usual Hartree-Fock-Bogolubov (HFB) approach to construct a quadratic Hamiltonian which accounts for the lowest order pairing. As before, we assume contact interactions and now make the additional assumption that we may neglect the excited modes of the molecular field keeping only the lowest condensed mode $\hat{b}_{\mathbf{k}}=b_{0}$. The resulting Hamiltonian for the composite system can be written in the form

$$
H=H^{0}+\sum_{\mathbf{k} \neq 0} A_{\mathbf{k}}^{\dagger} M_{\mathbf{k}} A_{\mathbf{k}}
$$

$H^{0}$ is composed of all terms of less then quadratic order in the operator $\hat{a}_{\mathbf{k}}$, we define a column vector $A_{\mathbf{k}}=$ $\left(\hat{a}_{\mathbf{k}}, \hat{a}_{-\mathbf{k}}^{\dagger}\right)$, and $M_{\mathbf{k}}$ is the self-energy matrix. For our purposes we need only concern ourselves with the structure of the second term in Eq. (10). Here the self-energy matrix is expressed as

$$
M_{\mathbf{k}}=\left(\begin{array}{cc}
E_{\mathbf{k}} & \Delta \\
\Delta^{*} & E_{-\mathbf{k}}
\end{array}\right)
$$

with the additional definitions for the diagonal and offdiagonal terms

$$
\begin{aligned}
E_{\mathbf{k}} & =E_{\mathbf{k}}^{0}+U\left(\left|\varphi_{a}\right|^{2}+n\right) \\
\Delta & =U\left(\varphi_{a}^{2}+p\right)+g \varphi_{m} .
\end{aligned}
$$

Equations (12) and (13) are expressed in terms of the pairing-field $p=\sum_{\mathbf{k}^{\prime}}\left\langle\hat{a}_{\mathbf{k}^{\prime}} \hat{a}_{-\mathbf{k}^{\prime}}\right\rangle$, the normal-field $n=$ $\sum_{\mathbf{k}^{\prime}}\left\langle\hat{a}_{\mathbf{k}^{\prime}}^{\dagger} \hat{a}_{\mathbf{k}^{\prime}}\right\rangle$, and $E_{\mathbf{k}}^{0}$ is the effective kinetic term which contains the contribution from the gauge field $A(\mathbf{x})$.

Eq. (10) can be rewritten in terms of the quasiparticles

$$
\begin{gathered}
\hat{\alpha}_{\mathbf{k}}=\frac{1}{\sqrt{\left(E_{\mathbf{k}}+\omega_{\mathbf{k}}\right)^{2}+|\Delta|^{2}}}\left(\left(E_{\mathbf{k}}+\omega_{\mathbf{k}}\right) \hat{a}_{\mathbf{k}}+\Delta \hat{a}_{-\mathbf{k}}^{\dagger}\right) \\
\hat{\alpha}_{-\mathbf{k}}^{\dagger}=\frac{1}{\sqrt{\left(E_{\mathbf{k}}+\omega_{\mathbf{k}}\right)^{2}+|\Delta|^{2}}}\left(\left(E_{\mathbf{k}}+\omega_{\mathbf{k}}\right) \hat{a}_{-\mathbf{k}}+\Delta^{\dagger} \hat{a}_{\mathbf{k}}\right)
\end{gathered}
$$

which result in the diagonal Hamiltonian

$$
\mathcal{H}=\mathcal{H}^{0}+\sum_{\mathbf{k} \neq 0} \omega_{\mathbf{k}} \hat{\alpha}_{\mathbf{k}}^{\dagger} \hat{\alpha}_{\mathbf{k}}
$$

where $\mathcal{H}^{0}$ contains the ground state contribution to the energy and the excitations are given by the spectrum of frequencies $\omega_{\mathbf{k}}=\sqrt{E_{\mathbf{k}}^{2}-|\Delta|^{2}}$.

Since there are no quasiparticles present in the ground state $|g s\rangle$, which is what one would expect from an interacting bosonic system at $T=0$, the ground state must satisfy the condition $\hat{\alpha}_{\mathbf{k}}|g s\rangle=0$. Substitution of Eqs. (14) and (15) for the quasiparticle operators result in the relation

$$
\left(E_{\mathbf{k}}+\omega_{\mathbf{k}}\right) \hat{a}_{\mathbf{k}}|g s\rangle=-\Delta \hat{a}_{-\mathbf{k}}^{\dagger}|g s\rangle .
$$

Because $\hat{a}_{\mathbf{k}}$ and $\hat{a}_{\mathbf{k}}^{\dagger}$ are canonically conjugate variables there is no loss of generality in making the replacement $\hat{a}_{\mathbf{k}} \rightarrow \partial / \partial \hat{a}_{\mathbf{k}}^{\dagger}$ [16]. This converts Eq. (17) into a simple differential equation for the ground state with the solution

$$
|g s\rangle=\exp \left[\sum_{\mathbf{k}} \frac{-\Delta}{E_{\mathbf{k}}+\omega_{\mathbf{k}}} \hat{a}_{-\mathbf{k}}^{\dagger} \hat{a}_{\mathbf{k}}^{\dagger}\right]|0\rangle .
$$

To derive the many-body wavefunction we must now move from second to first quantization. The relationship which links the second quantized ground state $|g s\rangle$ with the first quantized wavefunction $\Psi_{C B}$ can be written for an even number of noncondensed particles $2 N$ as

$$
\Psi_{C B}\left(\mathbf{x}_{1}, \mathbf{x}_{2}, \ldots \mathbf{x}_{2 N}\right)=\left\langle 0\left|\hat{\varphi}_{a}\left(\mathbf{x}_{2 N}\right) \ldots \hat{\varphi}_{a}\left(\mathbf{x}_{2}\right) \hat{\varphi}_{a}\left(\mathbf{x}_{1}\right)\right| g s\right\rangle,
$$

where it should be noted that $\Psi_{C B}$ is the full-many body wavefunction for the composite Bose particles. If we are able to assume that $E_{\mathbf{k}}+\omega_{\mathbf{k}} \gg \Delta$, an assumption which will remain valid as long as we are not too close to resonance, we may truncate the power expansion of the exponent in Eq. (18). This results in the composite boson wavefunction

$$
\Psi_{C B}\left(\mathbf{x}_{1}, \mathbf{x}_{2}, \ldots \mathbf{x}_{2 N}\right)=\mathcal{S}\left(\psi_{12} \psi_{34} \ldots \psi_{(2 N-1) 2 N}\right)
$$

comprised of a symmetrized product $\mathcal{S}$ of paired wavefunctions

$$
\psi_{i j}=\sum_{\mathbf{k}} \frac{-\Delta}{E_{\mathbf{k}}+\omega_{\mathbf{k}}} e^{i \mathbf{k} \cdot\left(\mathbf{x}_{i}-\mathbf{x}_{j}\right)} .
$$

If we were dealing with a system of fermions, equation (20) would be antisymmetrized and would result in a Pfaffian wavefunction [17]. Here, because of the statistics of the particles, we generate a bosonic analogue to this result. The many-body wavefunction for the bare particles can now be extracted from the composite wavefunction [18] resulting in 


$$
\Psi_{M B}=\Psi_{C B}\left(\mathbf{x}_{1}, \mathbf{x}_{2} \ldots, \mathbf{x}_{2 N}\right) \times \Psi_{L}\left(\mathbf{x}_{1}, \mathbf{x}_{2} \ldots, \mathbf{x}_{2 N}\right)
$$

which is a product of the composite particle wavefunction of Eq. (20) and the Laughlin wavefunction of Eq. (2).

Equation (22) is the final result for the ground state wavefunction of the resonant rotating Bose system. This result has important consequences for the generation of the FQHE within a resonant atomic gas. It would imply that an increase of interparticle interactions by a resonant tuning of the interactions simultaneously generates an increase in 2-particle correlations between composite particles resulting in a modification to the ground state wavefunction. As is clear from the form of Eq. (20), for large detuning from the resonance, corresponding to small pairing and molecular field, the many-body wavefunction reduces to the Laughlin wavefunction. As one moves nearer to the resonance, however, the off-diagonal part of the self-energy matrix, $\Delta$, grows. This results in an increasing modification of the many-body wavefunction from the Laughlin wavefunction. The ability to tune a Feshbach resonance therefore allows for the direct study of this crossover from a Laughlin wavefunction to a paired wavefunction.

A similar, yet distinct, paired wavefunction as in Eq. (22) was found for electronic FQHE systems 12, 17, 19]. This has been used to explain the previously unresolved even denominator filling fractions which result from a pairing instability, such as the observed incompressibility of the $5 / 2$ filling. In this case, a straightforward generalization of the Laughlin wavefunction would result in a symmetric wavefunction, violating the asymmetry of the fermions. However, the generation of an antisymmetric Pfaffian wavefunction which multiplies the generalized Laughlin state allows the overall ground state to be correctly antisymmetrized. For the bosonic system we have treated, the overall wavefunction must remain symmetric, so the corresponding paired wavefunction is symmetric in comparison to the antisymmetric Pfaffian wavefunction.

In conclusion, because of the extreme diluteness of trapped atomic gases, if strongly correlated effects such as the FQHE are to be observed in these experiments, the interatomic interactions will most likely need to be resonantly enhanced. Feshbach resonances are a convenient method for increasing the interactions, but the molecular processes involved in such a resonant system force one to account for the effects of atomic pairing. The growth of pair correlations among the composite particles leads to a modification of the expected ground state for a rapidly rotating Bose gas. The new ground state wavefunction, which is generated by the Feshbach resonance, exists as a strongly correlated state unique to trapped Bose gases. The tunability of the resonance opens the possibility for the direct study of the crossover transition between the paired state and the Laughlin state.

These results have important implications for the production of the FQHE within atomic gases since the use of a Feshbach resonance results in a state quite different from a rapidly rotating gas where the interactions are quantified by only a large scattering length. For instance, many of the observeable properties of the gas may be modified such as the density profile for both atoms and molecules and the nature of collective excitations. It should also be noted that the crossover transition we have discussed is only a part of a much more general crossover theory made accessible by the tunability of a Feshbach resonance. Although the methods presented here are invalid close to the resonance, one could imagine extending these ideas to describe the resonant system as one passes from a gas of interacting rotating atoms, through the resonance, to a system of tightly bound, rotating molecules.

Support is acknowledged for S.B., J.M., and M.H. from the U.S. Department of Energy, Office of Basic Energy Sciences via the Chemical Sciences, Geosciences and Biosciences Division.

[1] P. Engels, I. Coddington, P.C. Haljan, and E.A. Cornell, Phys. Rev. Lett. 89, 100403, (2002).

[2] J. R. Abo-Shaeer, C. Raman, J.M. Vogels, and W. Ketterle, Science 292, 476 (2001).

[3] T. L. Ho, Phys. Rev. Lett. 87, 060403 (2001).

[4] N. K. Wilkin, J. M. F. Gunn, and R. A. Smith Phys. Rev. Lett. 80, 2265 (1998).

[5] B. Paredes, P. Fedichev, J.I. Cirac, and P. Zoller, Phys. Rev. Lett. 87, 010402 (2001).

[6] U. R. Fischer and G. Baym, Phys. Rev. Lett. 90, 140402 (2003).

[7] S. L. Cornish, N. R. Claussen, J. L. Roberts, E. A. Cornell, and C. E. Wieman Phys. Rev. Lett. 85, 1795 (2000).

[8] T. Loftus, C. A. Regal, C. Ticknor, J. L. Bohn, and D. S. Jin Phys. Rev. Lett. 88, 173201 (2002).

[9] M. Holland, J. Park, and R. Walser, Phys. Rev. Lett. 86, 1915 (2001).

[10] E. Timmermans, P. Tommasini, R. Cote, M. Hussein, and A. Kerman, Phys. Rev. Lett. 83, 2691 (1999).

[11] T. Köhler and K. Burnett, Phys. Rev. A 65, 033601 (2002).

[12] F.D.M. Haldane and E.H. Rezayi, Phys. Rev. Lett. 60, 956 (1988).

[13] R.B. Laughlin, Phys. Rev. Lett. 50, 1395 (1983).

[14] S.C. Zhang, Int. J. Mod. Phys. B 6, 25 (1992).

[15] S. J. J. M. F. Kokkelmans, J. N. Milstein, M. L. Chiofalo, R. Walser, and M. J. Holland, Phys. Rev. A 65, 053617 (2002).

[16] P.A.M. Dirac, The Principles of Quantum Mechanics, (Clarendon Press, Oxford, 1958).

[17] G. Moore and N. Read, Nucl. Phys. B 360, 362 (1991).

[18] T. Morinari, Phys. Rev. B. 62, 15903 (2000).

[19] M. Greiter, X.G. Wen, and F. Wilczek, Nuc. Phys. B 374, 567 (1992) 